\title{
ANALYTIC CONTINUATION AND INFINITELY DIFFERENTIABLE FUNCTIONS
}

\section{S. MANDELBROJT}

It is our purpose to show that several branches of the theory of functions: detection of singularities, study of the behaviour of an analytic function in a strip or in an angle, a much more general theory than that of quasi-analyticity, convergence theorems, and so on, can all be based on a single principle, which may be regarded, in some ways, as a basic generalization of Cauchy's inequalities on Taylor coefficients.

This principle gives estimates of the $d_{n}$ 's in the series $\sum d_{n} e^{-\lambda_{n} s}$ by means of the maximum of the modulus of the function $F(s)$ it "represents." But the novelty of these evaluations lies in two essential facts: (1) It is supposed that the series represents the function in a certain horizontal strip-like region containing points with arbitrarily large abscissae, the series being, however, not supposed a priori to converge to the function anywhere. It has merely to "represent" the function with a certain "great precision," or, what amounts to the same thing, with a small error. (2) The evaluation is made by means of the maximum of the function in a circle which may lie as far to the left of the original region as the analytical continuation permits. The magnitude of the radius of this circle depends only on the distribution of the $\lambda_{n}$. The radius may be small if the $\lambda_{n}$ are sparse.

We have given some results of the same nature in papers published a few years ago, but our latest results - the general inequality and its applications to infinitely differentiable functions-seem to be of the "best possible" kind, since "nearly" converse theorems are also proved. At any rate, they are much more general than those we published in 1944.

Before we try to state the results clearly it is necessary to introduce some definitions.

Let $\left\{\lambda_{n}\right\}$ be an increasing sequence of positive numbers. We introduce the function $N(\lambda)=\sum \lambda_{n}<\lambda 1$, which is the number of $\lambda_{n}$ smaller than $\lambda$, the function $D(\lambda)=N(\lambda) / \lambda$-the density function, and $D^{*}(\lambda)=$ l.u.b. $x \geqq \lambda D(x)$ - the upper density function. The upper density function is obviously the smallest decreasing function larger than the density function.

An address delivered before the St. Louis meeting of the Society on November 29, 1947, by invitation of the Committee to Select Hour Speakers for Western Sectional Meetings; received by the editors November 22, 1947. 
The quantity $D^{*}=\lim \sup _{\lambda=\infty} D(\lambda)=\lim _{\lambda=\infty} D^{*}(\lambda)$ is called the upper density of $\left\{\lambda_{n}\right\}$. We shall suppose that $D^{*}<\infty$.

It will be convenient to introduce also the mean density function: $\bar{D}(\lambda)=\lambda^{-1} \int_{0}^{\lambda} D(x) d x$, the mean upper density function $\bar{D}^{\cdot}(\lambda)$ $=$ l.u.b. $x \geq \lambda \bar{D}(x)$, and the mean upper density of $\left\{\lambda_{n}\right\}: \bar{D}^{\cdot}=\lim$ sup $\bar{D}(\lambda)=\lim \bar{D}^{*}(\lambda)$. The inequality $\bar{D}^{\bullet} \leqq D^{\bullet}$ always holds, but examples show that the inequality $\bar{D}^{\bullet}<D^{\bullet}$ may actually hold.

Let $\Delta$ be a region in the $s=\sigma+i$ plane containing points with $\sigma$ positive and arbitrarily large. Let $F(s)$ be a function holomorphic in $\Delta$, and let $\left\{d_{n}\right\}$ be a sequence of real or complex numbers. We shall write $R_{m}(s)=F(s)-\sum_{1}^{m} d_{k} e^{-\lambda_{k s}}$. If $n$ is a given positive integer we say that $F(s)$ is represented in $\Delta$ by the set of Dirichlet polynomials $\sum_{1}^{m} d_{k} e^{-\lambda_{k s}}$, with $m \geqq n$, with logarithmic precision $p(\sigma)$, if for $s$ belonging to $\Delta$, and $x$ sufficiently large the inequality holds:

$$
\left.\underset{m \geq n}{\text { g.l.b. }} \underset{\sigma \geq x}{(1 . u . b .}\left|R_{m}(s)\right|\right) \leqq e^{-p(x)} \text {. }
$$

We shall always suppose that the logarithmic precision is a continuous function increasing to $+\infty$ (or eventually identically equal to $+\infty$ for $\sigma$ large).

If $x$ is sufficiently large, the difference between $\sum_{1}^{m} d_{k} e^{-\lambda_{k s}}$ and $F(s)$ can be made, in absolute value, smaller, in the part of $\Delta$ in which $\sigma \geqq x$, than any quantity $\delta>e^{-p(x)}$, provided $m$ (larger than $n$ ) is suitably chosen.

If the series converges to the function, the logarithmic precision can be taken equal to $+\infty$ for each $n>0$.

The converse is not generally true. But, if the logarithmic precision is equal to $+\infty$ (for $\sigma$ large) the series overconverges to $F(s)$ in $\Delta$.

A channel of width $2 R$ is the union of circles $C\left(s^{\prime}, R\right)\left(\left|s-s^{\prime}\right|<R\right)$, the radius $R$ being fixed, the centers $s^{\prime}$ taking all the values on a Jordan arc. The Jordan arc itself is the central line of the channel.

If a function $F(s)$ is holomorphic in the region composed of two regions $\Delta_{1}$ and $\Delta_{2}$, and a channel $C$ such that one of the circles of $C$ lies in $\Delta_{1}$, another in $\Delta_{2}$, we say that $F(s)$ can be continued analytically from $\Delta_{1}$ to $\Delta_{2}$ through the channel $C$.

The idea of the fundamental theorem is the following one:

If the sums $\sum_{1}^{m} d_{k} e^{-\lambda_{k}}(m \geqq n)$ represent $F(s)$, in a region sufficiently expansive (when measured vertically, for $\sigma$ large), with a sufficiently great logarithmic precision, and if the function can be continued analytically through a channel sufficiently wide to a circle $C\left(s_{0}, r\right)$, then $\left|d_{n}\right|$ is smaller than a certain quantity which depends on the following elements: (1) the abscissa of $s_{0},(2)$ the radius $r,(3)$ the 
maximum of $|F(s)|$ in $C\left(s_{0}, r\right),(4)$ the sequence $\left\{\lambda_{n}\right\}$ by means of the quantity

$$
\Lambda_{n}=\lambda_{n} \prod_{m \neq n} \frac{\lambda_{m}^{2}}{\left|\lambda_{n}^{2}-\lambda_{m}^{2}\right|}
$$

Let us now state the theorem rigorously:

FUndAMENTAL THEOREM. Let $\left\{\lambda_{n}\right\}$ be a positive increasing sequence with upper density $D^{\cdot}<\infty$. Let $\Delta$ be a region in the $s=\sigma+i t$ plane defined by: $\sigma>a,|t|<\pi g(\sigma)$ where $g(\sigma)$ is a function of bounded variation (in $[a, \infty)$ ), such that $g(\sigma)>D^{*}, \lim g(\sigma)>D^{*}$.

Let $F(s)$ be holomorphic in $\Delta$, and suppose that $F(s)$ can be continued analytically through a channel wider than $2 \pi D^{\circ}$ to a circle $C\left(s_{0}, \pi R\right)\left(R>D^{*}\right)$.

Suppose that the sequence $\left\{d_{k}\right\}$ is such that, for a given integer $n>0$, the Dirichlet polynomials $\sum_{1}^{m} d_{k} e^{-\lambda_{k s}}$ with $m \geqq n$ represent $F(s)$ in $\Delta$ with a logarithmic precision $p(\sigma)$ satisfying the relationship:

$$
\int^{\infty} p(\sigma) \exp \left[-2^{-1} \int^{\sigma}\left(g(u)-\bar{D}^{\cdot}(p(u))^{-1} d u\right] d \sigma=\infty .\right.
$$

Then

$$
\left|d_{n}\right| \leqq A(R) \Lambda_{n} M\left(s_{0}, R\right) \exp \left[\lambda_{n} \Re\left(s_{0}\right)\right]
$$

where $A(R)$ depends only on $R,{ }^{1}$ and where $M\left(s_{0}, R\right)=\max |F(s)|$ $\left(s \in \overline{C\left(s_{0}, \pi R\right)}\right)$.

In this theorem one may replace $D^{\bullet}$ by $\bar{D}^{\bullet}$ and $D^{\bullet}(\lambda)$ by $\bar{D}^{\bullet}(\lambda)$. If the sequence $\left\{\lambda_{n}\right\}$ is such that $\bar{D}^{\cdot}<D^{*}$, then with these replacements the hypotheses become less restrictive.

The condition (P) seems to be the most involved but as matter of fact, from a certain point of view it is, if not the best possible condition, at least an "almost best possible" one. We shall see the meaning of this assertion later on. But from now on we may notice that the theorem ceases to be true if, in (P), $D^{*}(p(\sigma))$ is replaced by its limit $D^{*}$ (such a condition would be less restrictive than (P)).

If the series $\sum d_{k} e^{-\lambda_{k s}}$ converges to $F(s)$ for $\sigma$ sufficiently large, then $p(\sigma) \equiv \infty$ (for $\sigma$ large) and the relationship (P) is satisfied automatically. In this case $g(\sigma)$ can be taken as large as possible, and the theorem is then reduced to the following statement:

If $\sum d_{k} e^{-\lambda_{k s}}$, with $D^{*}<\infty$, converges for $\sigma$ large to a function $F(s)$ which can be continued analytically, through a channel wider than $2 \pi D^{\circ}$,

${ }^{1}$ For a given sequence $\left\{\lambda_{n}\right\}$. 
to a circle $C\left(s_{0}, \pi R\right)\left(R>\bar{D}^{*}\right)$, then the evaluation (I) holds for each $n \geqq 1$.

Let us, for a moment, limit ourselves to the case in which the series converges to the function for large values of $\sigma\left(\sigma>\sigma_{c}\right)$. It follows then from the theorem that if $F(s)$ can be continued analytically from the half-plane of convergence, through channels wider than $2 \pi D^{\circ}$, to circles $C\left(s_{i}, \pi R\right)$ with $\lim _{i=\infty} \Re\left(s_{i}\right)=-\infty, F(s)$ being bounded in the set of these circles, then $F(s)$ is identically zero.

If, for instance, $F(s)$ is holomorphic and bounded in a strip $|t|<\pi C$ with $C>\bar{D}^{\cdot}$, then $F(s)$ is identically zero.

On introducing, moreover, the theory of normal families, we get the following result:

If the series $\sum d_{r} e^{-\lambda_{k s}}$ with $D^{\cdot}<\infty$ converges, for $\sigma$ large, to a function $F(s)$ non-identically zero, then in each strip $\Delta:|t|<\pi C$, with $C>\bar{D}^{*}, F(s)$ satisfies one of the three conditions:

(a) $F(s)$ admits there at least one singularity,

(b) $F(s)$ tends to infinity as $\lim \sigma \perp-\infty$ uniformly with respect to $t$, $s$ belonging to the strip $|t|<\pi(C-\epsilon)$, with $\epsilon$ arbitrarily such that $0<\epsilon<C$.

(c) $F(s)$ takes in $\Delta$ each value, except at most one, infinitely many times.

Many years ago, Ritt defined the order of a function given by a Dirichlet series convergent everywhere by:

$$
\rho_{R}=\limsup _{\sigma=-\infty} \frac{\log _{2} M(\sigma)}{-\sigma}
$$

where

$$
M(\sigma)=\underset{-\infty<t<\infty}{\text { l.u.b. }}|F(\sigma+i t)| .
$$

$F(s)$ being an integral function, let us set

$$
\begin{gathered}
M_{C}(\sigma)=\operatorname{liu.b.}_{|t|<x C}|F(\sigma+i t)|, \\
\rho_{R}(C)=\limsup _{\sigma=-\infty} \frac{\log _{2} M_{C}(\sigma)}{-\sigma} .
\end{gathered}
$$

We shall call $\rho_{R}$ the order $(R)$ of $F(s)$ in the plane, and $\rho_{R}(C)$ the order $(R)$ in the strip $|t|<\pi C$.

If $\lim \inf \left(\lambda_{n+1}-\lambda_{n}\right)=h>0$, then it is proved that

$$
\limsup _{n=\infty} \frac{\log \Lambda_{n}}{\lambda_{n}} \leqq A\left(h, D^{*}\right),
$$


where $A\left(h, D^{*}\right)$ is a quantity tending to zero, when $h$ is constant, $D^{*}$ tending to zero.

It is then easy to see from our general inequalities that if $C>\bar{D}^{*}$, $h>0$, then $\rho_{R}(C)=\rho_{R}$.

In other words: the order $(R)$ of a function given by a Dirichlet series convergent everywhere is, in each horizontal strip larger than $2 \pi \bar{D}^{\bullet}$, equal to its order $(R)$ in the whole plane.

This, together with a theorem proved by Bieberbach and Valiron (concerning the growth of functions in an angle), allows us to prove that if $D^{*}<\infty, h>0$, and if $\sum d_{k} e^{-\lambda_{k} s}$ represents an integral function $F(s)$ which is not a Dirichlet polynomial, then in each strip wider than $2 \pi \max \left(\bar{D}^{\circ},(2 \rho)^{-1}\right)$ there exists a straight line $t=t_{0}$ such that in each horizontal strip containing this line $F(s)$ takes each possible value, except at most one, infinitely many times.

With $\lambda_{n}$ integers and $\rho=\infty$, this result contains a theorem of Pólya. The line $\arg z=t_{0}$ is then a "line of Julia" of the function $\sum d_{k} z^{\lambda_{k}}$.

In a more restrictive form, these particular results concerning convergent Dirichlet series were already obtained by Gergen and myself many years ago.

On supposing still that the series admits an axis of convergence, let us see how the fundamental theorem gives results on singularities of functions represented by Dirichlet series. It follows from the general inequalities that if $\sigma_{C}$ is the abscissa of convergence of the series then

$$
\sigma_{C}=\limsup _{n=\infty} \frac{\log \left|d_{n}\right|}{\lambda_{n}} \leqq \limsup _{n=\infty} \frac{\log \Lambda_{n}}{\lambda_{n}}+\Re\left(s_{0}\right) .
$$

In other words, if $h>0$, and if $F(s)$ can be continued analytically through a channel wider than $2 \pi \bar{D}^{\bullet}$ to a circle $\left|s-s_{0}\right|<\pi R\left(R>\bar{D}^{\bullet}\right)$, then

$$
\Re\left(s_{0}\right) \geqq \sigma_{C}-A\left(h, D^{*}\right) .
$$

This leads immediately to a theorem of Ostrowski by which:

For each $h>0$ there exists a function $B_{h}\left(D^{*}\right)$, with $\lim _{D^{\cdot}-0} B_{h}\left(D^{*}\right)=0$, such that in each circle with center at $\sigma=\sigma_{C}$ and radius $B_{h}\left(D^{*}\right)$ the function $F(s)$ admits a singularity.

If, in particular, $D^{\cdot}=0$, we must have $\Re\left(s_{0}\right) \geqq \sigma_{C}$, the width of the channel being arbitrarily small. This shows that if $\lim n / \lambda_{n}=0(h>0)$, then each point on $\sigma=\sigma_{C}$ is a singular point. For Taylor series $\left(\lambda_{n}\right.$ integers) this is the famous Fabry's "gap theorem." For Dirichlet series it was proved by Szász. Other theorems on singularities can be obtained. 
Of course, the theorems on bounded functions in a strip or in a set of circles (theorems of Liouville's type), theorems of Weierstrass and Picard's type, still hold, if, instead of supposing that the series converges, we suppose merely that it represents the function in a region $\Delta$ (as in the fundamental theorem) with a sufficiently large logarithmic precision (the condition $(\mathrm{P})$ to be satisfied).

It may seem unnecessary to generalize results on convergent series to asymptotic ones. But we shall now show that our general theorem, in which it is not supposed that the series converges (instead, the condition $(\mathrm{P})$ is satisfied), gives results on infinitely differentiable functions, results which can be obtained only because the series is not supposed a priori to converge.

The link between the fundamental theorem and the theory of infinitely differentiable functions is given by a lemma which we shall state next. We must, however, first introduce a notion connected with infinitely differentiable functions.

If $f(x)$ is an infinitely differentiable function on the interval $I$ $(x \geqq 0)$, such that l.u.b. $x \geqq 0\left|f^{(n)}(x)\right|=m_{n}<\infty$, we shall call the function $C_{f}(\sigma)=$ l.u.b. $n \geq 1\left(n \sigma-\log m_{n}\right)$ the character of the function $f(x)$.

We prove the following lemma:

Let $f(x)$ be an infinitely differentiable function in $I(x \geqq 0)$ such that $\left|f^{(n)}(x)\right| \leqq M_{n}<\infty(n \geqq 0)$. Let $\left\{\nu_{n}\right\}$ be a sequence of non-negative integers such that

$$
f^{\left(v_{n}\right)}(0)=0
$$

Let $\left\{q_{n}\right\}$ be the sequence of non-negative integers complementary to $\left\{\nu_{n}\right\}$.

For each real a the function

$$
F_{a}(s)=\int_{0}^{\infty} \exp \left[-x e^{s-a}\right] f(x) d x
$$

is holomorphic in the region $\bar{\Delta}$ defined by $e^{\sigma} \cos t \geqq 1$. In this region the inequality holds: $\left|F_{a}(s)\right|<M_{0} e^{a} ;$ and for each $n \geqq 1, F_{a}(s)$ is represented in $\bar{\Delta}$ by $\sum_{1}^{m} d_{k}^{(a)} e^{-\lambda_{k s}}$ with $m \geqq n$, where $d_{k}^{(a)}=e^{\lambda_{k} a f\left(a_{k}\right)}(0), \lambda_{k}=q_{k}+1$, with the logarithmic precision equal to $C_{f}(\sigma-a)-a$, where $C_{f}(\sigma)$ is the character of $f(x)$.

It is clear that this lemma together with our fundamental theorem allows us to evaluate the derivatives of infinitely differentiable functions at the origin, if sufficiently many of these derivatives are supposed a priori to be zero (at the origin), and if the character of the function is sufficiently large. As a matter of fact, the evaluations so 
obtained depend on the quantity $a$ in such a way that they enable us to prove, by making $a$ tend to infinity, that all the derivatives are zero at the origin (if many of them are supposed to be zero, and if the character is large).

Here is the principal theorem concerning infinitely differentiable functions, obtained on combining the fundamental theorem with the lemma.

Let $f(x)$ be an infinitely differentiable function on $I(x \geqq 0)$, bounded with all its derivatives on $I$. Let $\left\{\nu_{n}\right\}$ be a sequence of non-negative integers with $\nu_{1}=0$, such that the upper density $D^{*}$ of its complementary sequence $\left\{q_{n}\right\}$ satisfies the inequality $D^{\bullet}<1 / 2$. Let $C_{f}(\sigma)$ denote the character of $f(x)$, and let $D^{\circ}(q)$ be the upper density function of $\left\{q_{n}\right\}$. If

$$
\int^{\infty} C_{f}(\sigma) \exp \left[-\int^{\sigma}\left(1-2 D^{\cdot}\left(C_{f}(u)\right)\right)^{-1} d u\right] d \sigma=\infty,
$$

and if $f^{\left(\nu_{k}\right)}(0)=0(k \geqq 1)$, then $f(x)$ is identically zero.

Of course, in this theorem, too, $D^{*}$ and $D^{*}(q)$ can be respectively replaced by $\bar{D}^{\bullet}$ and $\bar{D}^{\bullet}(q)$ (the mean upper density and the mean upper density function of $\left\{q_{n}\right\}$ ).

It is obvious that this theorem contains, as a very particular case, the classical theorem on quasi-analyticity. If, indeed, we suppose that all the derivatives are zero at the origin, that is to say, that the sequence $\left\{\nu_{n}\right\}$ is composed of all the non-negative integers, then $D^{\bullet}(q) \equiv 0$, and the theorem becomes:

If

$$
f^{(n)}(0)=0 \quad(n \geqq 0), \quad\left|f^{(n)}(x)\right| \leqq M_{n}<\infty \quad(n \geqq 0, x \geqq 0)
$$

and if

$$
\int^{\infty} C_{f}(\sigma) e^{-\sigma} d \sigma=\infty
$$

then $f(x) \equiv 0$.

In particular if $f^{(n)}(0)=0,\left|f^{(n)}(x)\right| \leqq M_{n}(n \geqq 0)$, and if, on setting

$$
A(\sigma)=\underset{n \geq 1}{\text { l.u.b. }}\left(n \sigma-\log M_{n}\right),
$$

we have:

$$
\int^{\infty} A(\sigma) e^{-\sigma} d \sigma=\infty,
$$


then $f(x) \equiv 0$. This is exactly another wording of the classical theorem (Denjoy-Carleman) on quasi-analyticity (Ostrowski's form).

However, not only does our theorem require merely $D^{\circ}<1 / 2$ in order to enable us to affirm that $f(x) \equiv 0$, if a suitable condition on $C_{f}(\sigma)$ is satisfied (condition $(Q)$ ), but, moreover, even if the classical condition is satisfied (condition $\left(Q^{\prime}\right)$ ) we do not need to suppose that all the derivatives are zero at the origin in order to conclude that $f(x) \equiv 0$.

For instance, the functions $f$ such that

$$
\left|f^{(n)}(x)\right|<K^{n}(n \log n)^{n}
$$

constitute a quasi-analytic class. By the classical theorem one can affirm that $f(x) \equiv 0$ only if it is supposed that $f^{(n)}(0)=0(n \geqq 0)$. But, since $C_{f}(\sigma)>C e^{\sigma} / \sigma$ we see, by our theorem, that if we suppose $f^{\left(\nu_{n}\right)}(0)=0 \quad\left(n \geqq 1, \nu_{1}=0\right)$, the complementary sequence $\left\{q_{n}\right\}$ being such that $q_{n}>a n \log n \log _{2} n$, we still may conclude that $f(x) \equiv 0$.

That the theorem on generalized quasi-analyticity cannot be much improved is shown by the following example: If in the just quoted example we replace the inequality $q_{n}>a n \log n \log _{2} n$ by $q_{n}>a n \log n$ then the assertion that $f(x) \equiv 0$ is not any more true.

As a matter of fact, the following theorem can be proved:

If the sequence of positive integers $\left\{q_{n}\right\}$ complementary to the sequence of non-negative integers $\left\{\nu_{n}\right\}\left(\nu_{1}=0\right)$ is such that $\lim \log q_{n} / n=0$, then there exists a function $f(x)$ not identically zero on $I(x \geqq 0)$, infinitely differentiable and bounded on I such that $f^{\left(v_{n}\right)}(0)=0$ and such that

$$
\left|f^{(n)}(x)\right| \leqq q_{1} q_{2} \cdots q_{n} \quad(n \geqq 1, x \geqq 0) .
$$

The proof of the fundamental theorem is difficult and technical and it would be impossible to indicate here even its main outlines. But it may be interesting to mention a theorem which plays an important role in the proof of the most general case.

The theorem generalizes a classical one which solves the Watson problem in a half-plane. As a matter of fact, this classical theorem served us as a tool when our statement of the fundamental theorem was less general.

Let $\Phi(s)$ be holomorphic in a region $\Delta$ given by $\sigma>a,|t|<G(\sigma)$, where $G(\sigma)$ is a function of bounded variation with $\lim G(\sigma)>0$, and let $\Phi(s)$ be continuous and bounded in $\bar{\Delta}$. Let $N(\sigma)$ be an increasing function.

If

$$
\log |\Phi(\sigma+i G(\sigma))|<-N(\sigma)
$$


and if on setting, for $d>a$,

$$
S(\sigma)=\frac{\pi}{2} \int_{d}^{\sigma} \frac{d u}{G(u)}
$$

we have

$$
\int^{\infty} N(\sigma) e^{-S(\sigma)} d \sigma=\infty,
$$

then the function $\Phi(s)$ is identically zero.

This theorem has its converse.

If, $N(\sigma)$ being an increasing function, $(\beta)$ does not hold, then there exists a function $\Phi(s)$, not identically zero, holomorphic and bounded in $\bar{\Delta}$ and such that $(\alpha)$ holds.

These two theorems were proved in a paper by G. R. MacLane and myself. If $G(\sigma) \equiv \pi / 2, a=-\infty$, the theorem gives the classical solution of a classical problem (generally stated for a half-plane $z=e^{s}$ ).

Let us mention that very recently we have proved jointly with Norbert Wiener results concerning infinitely differentiable functions of a character similar, if not easily comparable, to the theorem on the same subject stated above. The methods we used with Wiener are quite different from those I used to prove the theorem mentioned here. We employ an auxiliary function, holomorphic in the half-plane, with an argument corresponding to an order of differentiation, real or complex, with non-negative real part, and a value given by the value of the derivative of that order at the origin of a given infinitely differentiable function.

Let us notice that the fundamental theorem gives also conditions for convergence of Dirichlet series.

Suppose that $F(s)$ is holomorphic in a region described in the statement of the fundamental theorem, and let in this region

$$
\left|F(s)-\sum_{1}^{n} d_{k} e^{-\lambda_{k} s}\right| \leqq M_{n} e^{-\lambda_{n}} .
$$

It is then readily seen that for each integer $n, \sum_{1}^{m} d_{k} e^{-\lambda_{k} \varepsilon}$, with $m \geqq n$, represents $F(s)$ in $\Delta$ with the logarithmic precision equal to:

$$
p(\sigma)=\underset{n \geq 1}{\text { l.u.b. }}\left(\lambda_{n} \sigma-\log M_{n}\right) \text {. }
$$

Therefore, if, with the notations just introduced, $(P)$ holds, then 
(I) holds for each $n>0$. It can then be easily shown that if (C) and (P) hold (with $p(\sigma)$ defined by $(\mathrm{M})$ ), and if $h>0$, then $\sum d_{k} e^{-\lambda_{k s}}$ converges to $F(s)$ for $\sigma$ sufficiently large.

\section{BIBLIOGRAPHY}

S. Mandelbrojt, Quasi-analyticity and analytic continuation-a general principle, Trans. Amer. Math. Soc. vol. 55 (1944) pp. 96-131.

352-378.

S. Mandelbrojt and G. R. MacLane, On functions holomorphic in a strip, and an extension of Watson's problem, Trans. Amer. Math. Soc. vol. 61 (1947) pp. 457-467.

S. Mandelbrojt and Norbert Wiener, Sur les fonctions indéfiniment derivables sur une demi-droite, C. R. Acad. Sci. Paris vol. 225 (1947) pp. 978-980.

- Quasi-analyticité générale et theorèmes du type Phragmén-Lindelöf, C. R. Acad. Sci Paris vol. 226 (1947) pp. 47-49.

Collège de France and Rice Institute 